\title{
Electrical Conductivity of a Precipitate from a Sulfuric Acid Solution of Poly(acetylene)
}

\author{
Seizo Miyata, Shigeru TASAKA, Yoshitomo MaSUdA, \\ and Munam KO* \\ Faculty of Technology, Tokyo University of Agriculture and Technology, \\ Nakamachi, Koganei, Tokyo 184, Japan \\ *Faculty of Science, Korea University, \\ Ogawa-cho, Kodaira, Tokyo 187, Japan
}

(Received April 12, 1983)

\author{
KEY WORDS Poly(acetylene) / Electrical Conductivity / Solvent / Sulfuric \\ Acid /
}

Since the high electrical conductivity of doped poly(acetylene) was discovered by Shirakawa et al., ${ }^{1}$ the conductivity of this material has been extensively investigated. However, in spite of the considerable research that has been carried out on its solid state physical properties, polyacetylene has been poorly characterized. Most of the difficulties in this regard arise from the lack of appropriate solvents for poly(acetylene). If a suitable solvent is available, both its structure and properties can be elucidated and the processing techniques for obtaining its films and fibers developed. We report here our attempts at finding a liquid poly(acetylene).

Poly(acetylene) powder was prepared by polymerizing acetylene with a Ziegler-Natta catalyst at dry ice methanol temperature. ${ }^{2}$ The samples used for electrical conductivity measurements were prepared by compression molding of the polyacetylene powder into disks approximately $0.1-0.2 \mathrm{~mm}$ in thickness. Platinum wires and graphite paste were used to obtain electrical contacts with the disks. The disks were treated with iodine vapor at room temperature. The conductivity was monitored at suitable intervals of time. The composition of the doped poly(acetylene) disks was determined from the increase in weight of the disks during application of the four-point probe method.

When poly(acetylene) was heated in concentrated sulfuric acid, it become soluble at $150^{\circ} \mathrm{C}$ and completely dissolved at a temperature higher than $200^{\circ} \mathrm{C}$, forming a homogeneous black solution. The solution so obtained did not give any precipitate even after cooled to room temperature. However, when the solution was poured into water, aceton or methanol, poly(acetylene) began to coagulate and precipitate.

Figure 1 shows the electrical conductivity at room temperature for both the original and precipitated samples as a function of iodine concentration. At zero iodine concentration, the electrical conductivity of the precipitated soluble sample was nearly the same as that of the original poly(acetylene). However, the rate of its increase with doping differed in these two cases. Following an initial steep rise exceeding about four orders of magnitude, the electrical conductivity of the precipitated sample appeared to saturate at $4 \times 10^{-4} \Omega^{-1} \mathrm{~cm}^{-1}$, while that of the original poly(acetylene) leveled off at $5 \Omega^{-1} \mathrm{~cm}^{-1}$. The latter was rather low compared with the literature value ${ }^{1}$ possibly since our measurements were carried out with disks obtained by compressing powder samples and having higher contact resistance.

Thus, although the electrical conductivity leveled off at a lower value than that of the original poly(acetylene) the sample precipitated from the concentrated sulfuric acid solution showed doping effects, indicating that conjugated double bonds were retained in the molecule. The black color of the sample and the broad absorption spectrum in the 


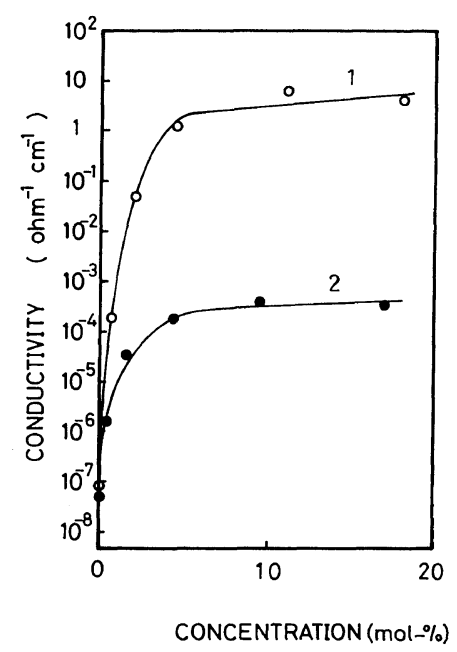

Figure 1. Conductivity as a function of dopant concentration: (1) original polyacetylene; (2) precipitate from a sulfuric acid solution.

visible region may be regarded as evidence for the $\pi \rightarrow \pi^{*}$ transition between the most occupied and the least vacant orbitals in the polymer molecule.

The precipitated samples gave broad rather than sharp infrared spectra. However, a large peak was observed in the vicinity of $1700 \mathrm{~cm}^{-1}$, indicating the existence of $\mathrm{C}=\mathrm{O}$ groups. These carbonyl groups possibly formed by oxidation with sulfuric acid at high temperature limit the conjugation of double bonds to shorter sequences. However, some undegraded chains should remain in the system and cause the observed increase in conductivity with doping.

Since sulfuric acid changes the chemical structure of poly(acetylene), it cannot be considered a true solvent but its action on chemical structure should provide a guide for finding a suitable solvent. We also found that fumic sulfuric acid dissolves poly(acetylene) even at a temperature lower than that when using sulfuric acid. Probably, other strong acids such as fluorosulfuric acid may be capable of dissolving poly(acetylene).

Acknowledgment. The authors wish to express their appreciation to Professor Hideki Shirakawa of Tsukuba University for his useful comments.

\section{REFERENCES}

1. H. Shirakawa, E. J. Louis, A. G. MacDiamond, C. K. Chiang, and A. J. Heegar, J. Chem. Soc., Chem. Commun., 578 (1977).

2. G. Natta, G. Mazzanti, and P. Corradini, Rend. Accad. Naz. Lincei, 25, 3 (1958). 